\title{
UNDERLYING ITS BUSINESS PROCESSES WITH FLEXIBLE AND PLUGGED PEER SYSTEMS: THE OPEN ITS-IBUS APPROACH
}

\author{
A. Luís Osório, M. Martins Barata, Arnaldo J. Abrantes, \\ ISEL, Instituto Superior de Engenharia de Lisboa, DEETC department, Lisboa, PORTUGAL \\ J. Sales Gomes, Gastão C. Jacquet \\ BRISA, Auto-estradas de Portugal, DID Innovation and Development Department, \\ Carcavelos, PORTUGAL
}

\begin{abstract}
The extension of the Via-Verde business model from motorways and bridges to car parking and gas stations involving different enterprises has motivated the proposal of an open integration bus to answer the new challenges for systems integration. It is discussed a service framework based on a peer to peer distributed architecture where peers offer specialized services contributing to the execution of virtual business processes. Those specialized services are executed in a system container with "plug and play" features and some of them assume the responsibility for the execution and coordination of the business processes. The paper discusses the underlying strategy that has motivated BRISA, as ITS end-user, to promote an open strategy for the ITS-IBus integration framework. It is also discussed a strategy based on a distributed service framework offering an added flexibility to virtual business process execution and coordination.
\end{abstract}

\section{INTRODUCTION}

The Intelligent Transport Systems (ITS) area is facing emerging new business models based on complex partnerships among pervasive enterprises, requiring new approaches to the underlying technological base. Furthermore, a trend for a world of services, as discussed in (Eriksson, 2002), is motivated by an increased importance of some new facilitators like cellular phones, PDAs, wireless telecommunication, Global Positioning Systems (GPS) and geographic information systems (GIS). Main ITS visions point to the emergence of virtual organizations requiring a new technological architecture able to execute the complex collaborative business processes (ITS/EC, 2002). The ITS-IBus (ITS Integration Bus) project was motivated by the BRISA's need to rethink toll motorway technology management in order to reduce systems lifecycle costs associated to an increased responsiveness with an added service quality. The new virtual payment system extends the toll fee collection infrastructure to car parking and gas stations and it has created interoperability challenges which are difficult to overcome with existing technological frameworks. The offered "quality of services and coordination mechanisms are not compatible with the new dynamics of the cooperative business 
process. The adopted strategy involves the promotion of an open initiative around an Intelligent Transport Systems Integration Bus (ITS-IBus). It aims to contribute to an advanced framework able to cope with the new challenges grounded on a generalized cooperation among distributed and multicultural enterprises. The proposed ITS-IBus considers basically two approaches: a bottom-up and a top-down strategy. The bottom-up approach considers an extended effort that is being done to establish the concept of plugged peer system based on existing systems and standards (Osório 2003). The project is working on the DSRC (Dedicated Short Range Communication) and on automatic license plate recognition (LPR) systems as case studies to demonstrate the proposed interfaces and associated behaviors. The top-down approach is focused on the architectural issues considering the distributed business processes execution and coordination requirements. This view is mainly concerned with the establishment of a framework coping with distributed process (services) design and execution requirements considering that they are spread among contributing enterprises, those that are part of the cooperative and distributed business processes: the virtual organization members.

This paper discusses ITS-IBus architectural perspective considering the underlying peer systems as plugged and flexible service providers. The virtual business process requirements are also discussed considering aspects like coordination, availability, security and systems life cycle management.

\section{MOTIVATIONS FOR THE ITS-IBUS AND VISION}

Enterprises are facing new challenges when deciding about a number of information and communication technology (ICT) facilitators able to contribute to automate their business processes. In fact the last technological evolutions, mainly those related to Internet, have created some over expectations resulting from the lack of a clear and solid architecture able to deal with the underlying complexity. Since the end of the last century different works have claimed for architectures crossing enterprise borders. This move from an enterprise centered analysis where several models have been discussed like CIMOSA (Vernadat, 1996) to an enterprise network centered view, have established the importance of the virtual organization perspective. According to Byrne "A Virtual Corporation is a temporary network of independent companies - suppliers, customers, even competitors - linked by information technology (IT) to share skills, costs and access to one another's markets. It will have neither central office nor organization chart. It will have no hierarchy, no vertical integration" (Byrne, 93). On the other hand in (Camarinha, 1999) this and other definitions are synthesized by stating a virtual enterprise as a "temporary alliance of enterprises that come together to share skills or core competencies and resources in order to better respond to business opportunities and whose cooperation is supported by computer networks". Another view from the management area establishes organization virtualness when work deliverables receive contributions from different locations, different work cycles and across cultures, following six virtual models: virtual faces, co-alliance, value-alliance, star-alliance, marketalliance and virtual broker (Burn, 1999).

In concurrence with these more academic discussions about virtual enterprise models several businesses emerged and are now the support for daily life citizen 
services across the entire world. One example of such a business model crossing different enterprises is the "Via Verde" toll collection system implemented by BRISA company and now managed by Via-Verde-Portugal consortium. The cars holding an identifier based on DSRC technology have the opportunity to extend ViaVerde services to a non-stop car parking access and to fill their car gas tanks without leaving the pump area. This virtual business model has created new challenges to both organizational and technological levels. In this direction our first effort was directed to discuss technological issues and was based on a key question posed by BRISA: how to answer to the crescent complexity to manage pervasive and heterogeneous systems that in conjunction underlie Via-Verde business model? It was established the need for an integration framework based on open interfaces for the involved supporting subsystems (DSRC, parking, gas stations).

There is another challenge related to process integration at business model level. The Via-Verde business model involves several companies each one with its own interests but all cooperating to a common business. The involved companies (BRISA, VVP, BER, ACCESS from others) have complementary responsibilities (deliverables), different technologies and process cultures but agreed on a common business model with an underlying business plan made of shared business processes. This required a new strategy not only to cope with distributed business process management and coordination but also the establishment of a common integration strategy able to promote interoperability among the involved diversity of technological solutions. To that end the ITS-IBus open initiative has established a service framework based on fine grained functional entities organized as specialized services. These services establish a common distributed execution infrastructure based on a set of standard interfaces and protocols. The legated monolithic systems have suffered a "disintegration" process and were redesigned based on a set of services presenting open interfaces as discussed in (Gomes, 2003), (Figure 1).

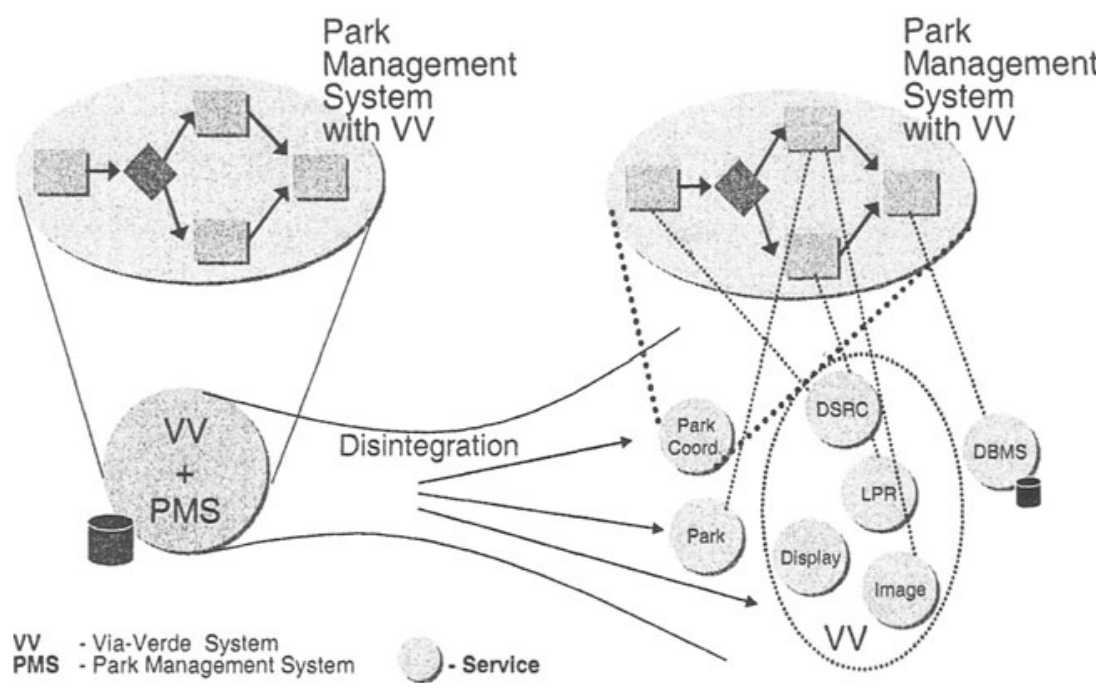

Figure 1 - The disintegration process of monolithic systems (enterprise applications) 
The ITS-IBus project, started from the hypothesis that the development of an open framework where different service implementers can be plugged, offers an increased flexibility, a reduced system lifecycle management costs and contribute for a more competitive ICT (Information and Communication Technology) industry. The required flexibility involves the two above mentioned views, on the one hand an easy adoption of interface implementations from different suppliers and, on the other hand, coping with (virtual) process requirements. The strategy considers the shift from a monolithic approach towards a fine grained service infrastructure applied to the virtual organization level where business plans are shared among different enterprises (Figure 2). These virtual business processes (VBP) require well established management and coordination policies. The distribution associated to the multicultural aspect adds a new complexity dimension to the required holistic approach to Via-Verde business model. A well established management and coordination for the local deliverable to the VBP is also a fundamental commitment for each participating enterprise.

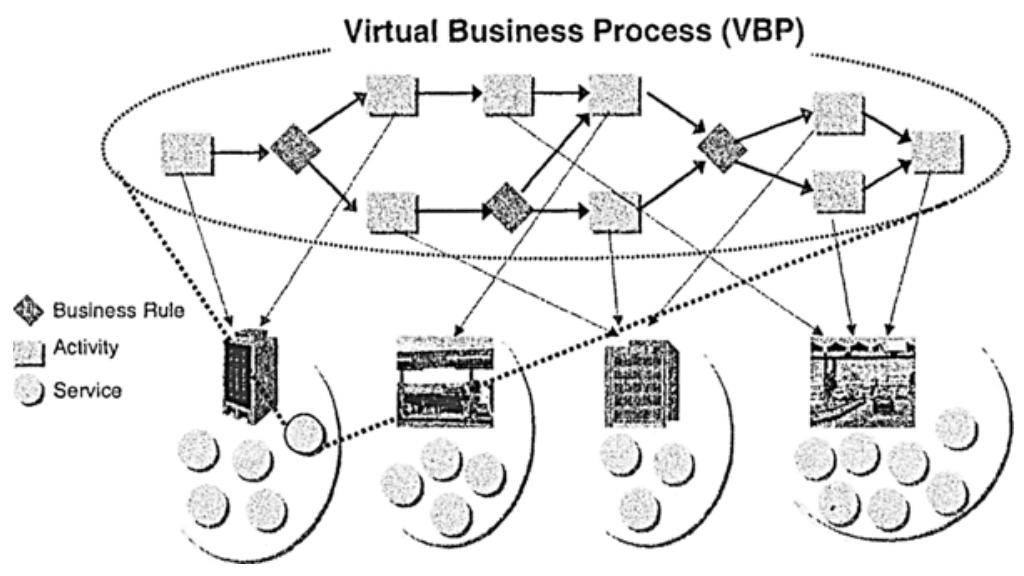

Figure 2 - A Virtual Business process "executed" by a virtual organization

Therefore the ITS-IBus is more than a simple middleware communication framework, it aims to be a consensus framework able to deal with several business processes receiving contributes from different enterprises. There are some more economical related reasons for a company like BRISA to bet on the establishment of a pluggable framework where systems can be integrated following the "plug and play" concept already well established for the Windows operating system. It is expected to get lower costs, more evolving, more scalable and safe technological infrastructure for BRISA and for other end-users.

Assuming a global process plan representing the set of cooperative or (virtual) business processes responsible for specific aspects of the toll related business, the ITS-IBus proposes to establish an open integration bus able to logically connect all the contributors based on the service paradigm. The openness perspective follows other initiatives promoted by end-users towards the definition of common concepts and specifications. When promoted by market weight end-users these specifications 
are expected to contribute to promote competitiveness including among small and medium companies (SME). They can base the required market confidence on the implementation of standards reducing the risks that potential customers usually see on small companies. These weaknesses presented by the SME companies can be minimized if their contributions follow standards considering that their participation as suppliers can be more easily replaced if by some reason they can not cope with some settled commitment.

\section{EVOLVING FROM CLIENT-SERVER TO PEER TO PEER ARCHITECTURES}

The client-server models present weaknesses when coping with the required holist approach to virtual business process automation. They rely on centralized servers behaving as single point of failure what makes difficult to offer reliability to virtual business process execution. Furthermore, existing enterprise integration models rely on the definition of partial functionalities organized around a hierarchy of heterogeneous and heavy execution systems difficult to integrate. The "glue" of these enterprise information systems (EIS) or more broadly, enterprise ICT (Information and Communication Technologies) systems is based on a "one to one" integration following the request-reply model synchronous or asynchronous, in most of the cases, difficult to maintain and evolve considering the complex transactions. Another drawback from this approach is the low level reutilization and in many cases, functionalities and information entities are replicated by several enterprise ICT systems (Gomes, 2003). The workflow systems have contributed to create some flexibility on business process execution and coordination but considering that the WFMC (Work Flow Management Coalition) architecture deeply rely on the clientserver model addressing partial integration areas like enterprise document and content management it also presents limitations to a more holistic and flexible approach.

There is a crescent discussion about other organization forms and based on our research it is necessary a rupture with the approach ICT engineering and other disciplines are following to model ICT technologies. It is also necessary to define new methodologies associated to the life cycle management of the virtual business processes and underlying service based framework. The peer to peer concept also discussed in the context of the distributed database management systems (Frenkel, 2000) is an important issue for the peer to peer distributed services. As discussed by (Risse, 2003) services might return objects, they might be passed to other services, at the end there is a need for persistence in order to maintain distributed information consistence considering the application (or the virtual business process requirements).

According to the peer to peer working group (www.peer-to-peerwg.org) a peer to peer (P2P) distributed architecture involves "the sharing of computer resources and services by direct exchange between systems. These resources and services include the exchange of information, processing cycles, cache storage, and disk storage for files". The P2P architectures have received a crescent interest with some successful initiatives and projects like Napster focused on resource sharing, SETI* (Search for Extraterrestrial Intelligence*) aiming to provide distributed parallel computing for 
computational intensive processes and Grove developed to support collaborative based applications. All these P2P systems consider a huge number of users or peer systems and in some cases there are no centralized services. A pure P2P architecture rely on distributed equal execution entities able to offer specialized services made known through advertisements possibly crossing firewalls and routing/NAT (Network Address Translation) barriers (Figure 3).

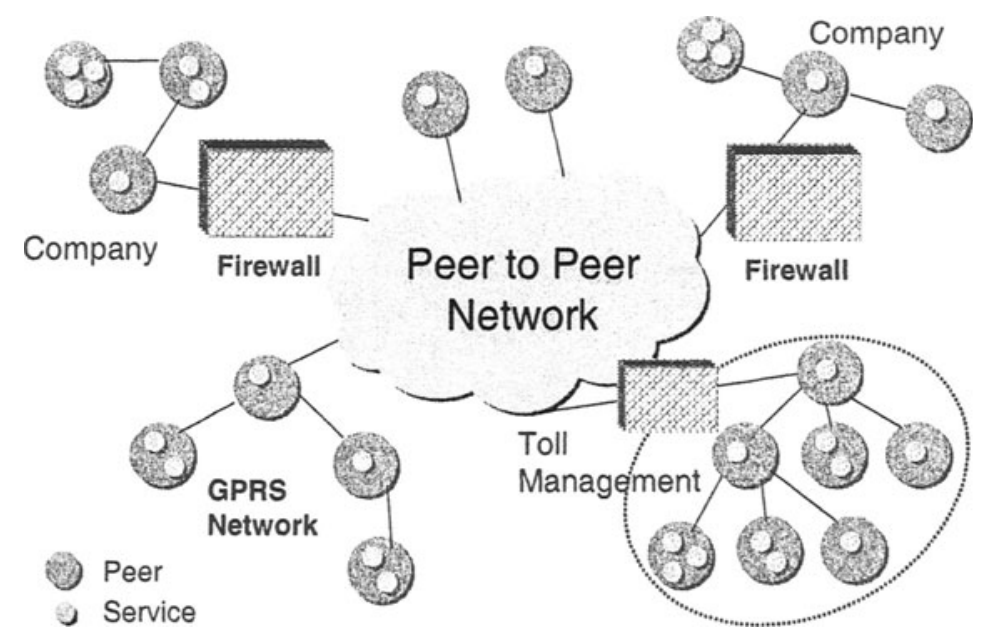

Figure 3 - An example of a network of peers

The JXTA initiative (Brendon, 2002), (Govoni, 2002) has been developing an open framework offering a development and execution framework to develop peers and peer systems based on a set of core structuring concepts:

- Peers are organized into groups establishing shared communication spaces limited to group members. A peer can communicate directly to each other or they can multicast a message to all the group members. All the peers belong to the NetPeerGroup world root group;

- The framework does not rely on centralized services. A set of protocols provide discovery and advertisement mechanisms for the available services;

- It is platform independent considering languages, transport protocols and deployment platforms. It can be considered a platform independent model (PIM) according OMG definition (Siegel, 2001);

- The ubiquity characteristic is associated to its development and deployability to platforms from PC, PDA, mobile phones and other dedicated systems;

- Beyond a set of standard and community services the JXTA offers a set of core services common to all peers. These services involve security, pipes and peer monitoring.

The JXTA does not include "plug and play" mechanisms for the peers. However, this functionality can be supported as a new service or supported in the peer advertisement itself. The "plug and play" concept definition and reference 
implementation have been conducted by the UPnP consortium which is mainly focuses on home and small office devices/systems application (Miller, 2001).

There are some advantages on adopting peer to peer architectures when comparing to the classical client server paradigm. Even if P2P architectures might present more failure points considering peer reach-ability, strategies like service replication can contribute to flexible and reliable systems. Nevertheless new challenges are presented by the P2P architectures. For a large number of peers the communication latency might get unacceptable values. As an example, a lookup for a specific service through its advertisement may take a long time depending on the dimension and extensibility of the peer network. For a network of peers based on Internet the scale problems might be evidenced. Concerning security JXTA adopt the "web of trust" paradigm, where peer authentication is based on trusted groups (Fox, 2001) and links among them. In web-of-trust peers decide about the other peers they trust with (Caronni, 2000). Communication can be done through secure communication pipes in order to guarantee privacy during message exchange.

Even if the JXTA approach can be considered a platform independent model (PIM), the available reference implementations are deeply compromised with Java world. Furthermore, there are some contributions arguing in favor of the native characteristics of Microsoft .NET framework to support peer to peer computing scenarios like the simple publish/subscribe implementation proposed by (Dasgupta, 2001). The proximity of the .NET to the Web service paradigm is a key argument in favor to its proximity to a peer to peer architecture. There is however a lacking of some grounding concepts, like the group or hierarchy of groups to establish peer with common characteristics or peer contexts.

The ITS-IBus project has established a PIM approach and considers the development of a reference implementation based on JXTA or the.NET frameworks.

\section{THE ITS-IBUS AS A SERVICE BASED FRAMEWORK}

The ITS-IBus architecture relies on the assumption that ITS systems involve a huge number of distributed and multiple enterprise heterogeneous ICT systems. Each ITS system has an underlying business plan organized around a hierarchy of business processes where each one represents a group of activities governed by a set of business rules (Osório, 1998), (Gomes, 2003). The business process management institute (BPMI) was created to develop, publish, maintain, and promote a common meta-language (BPML) to support process design, deployment, execution, maintenance, and optimization to manage business activities along their entire lifecycle. The web services are becoming a point of convergence for the software systems engineering industry and in concurrence to BPMI other initiatives are addressing business process representation. The Web Services Choreography Interface (WSCI) promoted by W3C through the web services choreography Working Group aims to define a language for business process (Trickovic, 2003), in the line of the BPMI and BPELAWS this proposed by IBM, Microsoft and Bea. The workflow management coalition WFMC's XML process definition language (XPDL) and the OASIS's ebXML BPSS are also initiatives to be considered for business process life cycle representation. 
The ITS-IBus underlying strategy considers the virtual enterprise applications or enterprise subsystems as a group of services that contribute to the execution of some (virtual) business process. A business process receives contributions from services in a company or, if a virtual business process, contributions from services running in different companies. As shown in (Figure 1) the park coordinator is a service responsible for the execution of the business process associated to a car parking management system under Via-Verde business model (Gomes, 2003). The services that contribute to some business process or enterprise application are "glued" through the ITS-IBus which can be based on a single physical communication bus or, as it happens in a toll lane, it might involve different networks in order to cope with real-time constraints (Figure 4). A Via-Verde lane also needs to answer car detection, classification and identification through automatic plate recognition (LPR) for the case enforcement is needed.

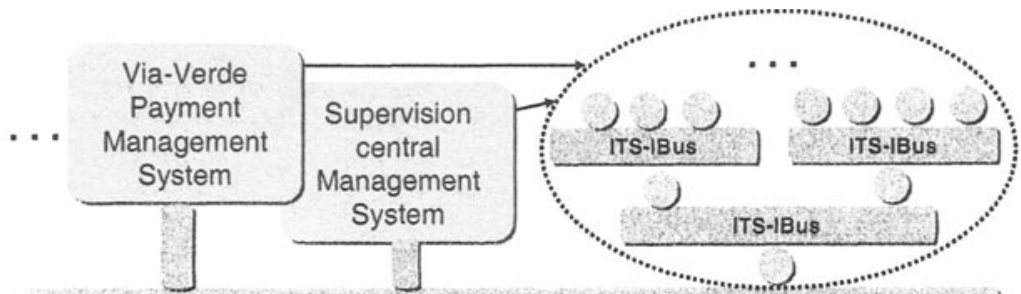

ITSIBus - Intelligent Transport Systems Integration Bus

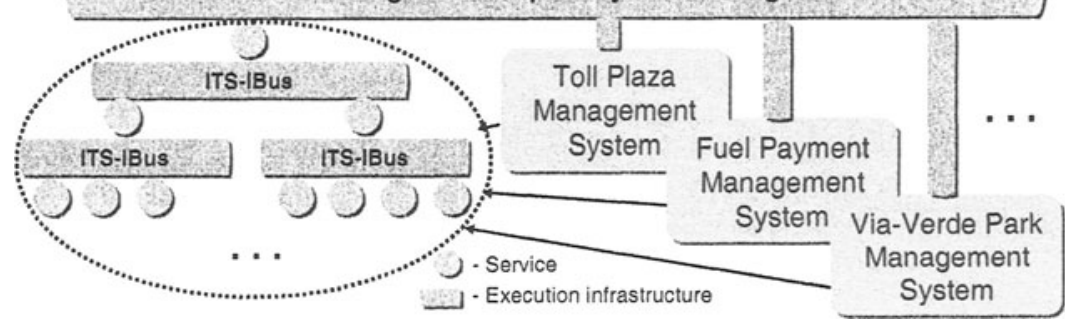

Figure 4 - The ITS-IBus framework based on services

Therefore ITS-IBus has adopted a peer to peer strategy considering that business processes are executed into systems that present services to other processes or service implementations (Figure 5). Everything requiring computational actions is represented by services presenting an open interface and following a set of rules that make them ITS-IBus enabled. The services are plugged into systems that behave like an execution containers presenting a minimal set of service to the ITS-IBus (Gomes, 2003). Plugged services are registered to systems and can use devices to implement the exported operations. A system might implement at least the plug and play service in order to be identified by other ITS-IBus enabled peers.

Services are implemented in different language and using different execution environment (Figure 5) considering operating systems (Windows, Linux or other) and distributed infrastructures (CORBA, Java/RMI or .NET Remoting). The proposal points to lightweight execution entities based on a spatial distributed fine granularity able to cope with a wide range of business process requirements. This opposites the classical approach where heavy ICT systems (also known as enterprise 
information systems or enterprise applications) address specific enterprise process domains and embed the business process logic "hard-coded" and under a proprietary strategy. This heavy "gross grained" business process automation does not promote reutilization and creates difficulties to offer holistic enterprise business process views. Furthermore, it is not able to cope with business process automation at virtual organization level.

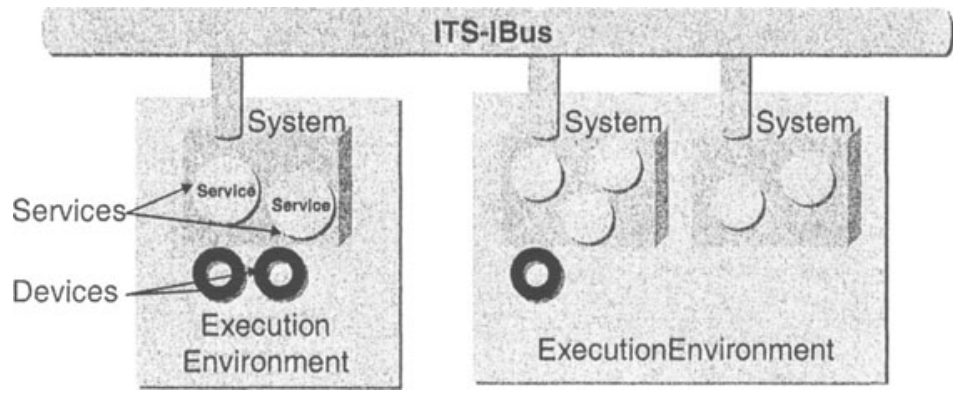

Figure 5 - The ITS-IBus execution unit organization strategy

A first prototype to model toll plaza business processes is being developed in order to consolidate the ITS-IBus concept. This work considers the Business process plan as a global meta-structure modeling the business processes that are somehow related to contribute to a common business objective (Osório, 1998). The development of a language representation for the business processes will not be considered for a first approach to prototype developments. The underlying business logic associated to the under construction demonstrators will be hard-coded even if more research will be done to decide for a business language representation strategy.

A lane controller has the responsibility to coordinate the devices that support car toll payment. Furthermore, the lane controllers are coordinated at a toll plaza level by a toll plaza controller. It makes use of the services of lane type peers to implement plaza controller business process and, in addition, exposes to ITS-IBus a set of services that will be used by the peer that implements central toll management infrastructure, the central toll business management.

\section{CONCLUSIONS}

A Peer to peer or web service based architecture presented as service framework was discussed as a strategy to underlie a flexible integration framework for ITS technological systems. The discussed framework is based on a flexible representation and execution of the enterprise processes where activities and business process coordination rely on a set of services bound into systems (peers). The motorway toll management and a car parking systems both considering the ViaVerde business model where car drivers are able to cross motorway toll lanes and access car parking infrastructures using the same payment service, are being used to demonstrate the ITS-IBus proposed concepts and strategies. 
This work was motivated by the challenges to extend Via-Verde business model to car parking and gas station services. The Via-Verde was initially adopted to motorways and bridges and even involving different enterprises (operators) the technological systems were similar. However with the extension to parks and gas stations the diversity of the involved technological systems and the diversity of the specific business processes from each participating company required a new approach to this new virtual organization model.

\section{ACKNOWLEDGEMENTS}

This work was partially supported by BRISA group, through the research and development BRISA-PARK and ITS-IBus projects. The work is being developed by the research groups GIATSI and Signals Processing groups from ISEL-DEETC in collaboration with DID/NID, the Innovation and Development Department of BRISA. We also acknowledge the valuable contributions from Diogo Remédios, Filipe Braz, Bruno Basílio and Rui Lopes.

\section{REFERENCESS}

1. Brendon J. Wilson - JXTA, New Riders Publishing; ISBN: 0735712344, 1st edition, June 15, 2002.

2. Camarinha-Matos, L. M., Afsarmanesh, H., - The Virtual Enterprise Concept, in Infrastructures for Virtual Enterpriuses, Klwer Academic, ISBN 0-7923-8639-6, cap. 1 part I, pp. 3-14, 1999.

3. Caronni G. - Walking the Web of Trust, proceedings of the 9th Workshop on Enabling Technologies (WET ICE'2000), IEEE Computer Society Press, 2000.

4. Dasgupta, Rana - .NET as a Development Platform for Peer-to-Peer Applications, Information for Developers and ISVs, Intel Corporation 2001.

5. Eriksson O. - Intelligent Transport Systems and Services (ITS) - New challenges for system developers and researchers, International Conference of IS Development, Riga, Latvia September, 2002.

6. Fox, F.; Gannon, D. - Computational grids, IEEE Computational Science and Engineering, Volume: 3 Issue: 4, Jul/Aug 2001.

7. Frenkel A., Afsarmanesh H., Garita C., Hertzberger L. O. - Support Information Access Rights and Visibility Levels in Virtual Enterprises, PROVE-2000 E-Business and Virtual Enterprises (Kluwer Academic), Florianópilis Brasil, December 4-6, 2000.

8. Gomes J. Sales, Jacquet G., Machado M, Osório A. Luís, Gonçalves C., Barata M. - An Open Integration Bus for EFC: The ITS IBus, ASECAP2003, May 2003 in Portoroz, Slovenia.

9. Govoni D., Krishnan N., Soto J. C. - JXTA: Java P2P Programming, Sams; ISBN: 0672323664, 1st edition, March 22, 2002.

10. ITS/EC - ITS/European Commission, European Standardization in Intelligent Transport Systems - A proposed European programme, CMC/ISSS, August 2002.

11. Miller, B.A.; Nixon, T.; Tai, C.; Wood, M.D.; Home networking with Universal Plug and Play, IEEE Communications Magazine, Volume: 39 Issue: 12, Dec 2001.

12. Osório A. L., Abrantes A. J., Gonçalves J. C., Araújo A.; Miguel J. M., Jacquet, G. C.; Gomes, J. S. Flexible and Plugged Peer Systems Integration to ITS-IBUS: the case of EFC and LPR Systems, PROVE'03 - $4^{\text {th }}$ IFIP Working Conference on Virtual Enterprises, 2003.

13. Osório A. L., Oliveira N., Camarinha-Matos L.M. - Concurrent Engineering in Virtual Enterprises: The extended CIM-FACE architecture, Proc. Of BASYS'98, Intelligent Systems for Manufacturing (Kluwer Academic), Prague, Czech Republic, August 1998.

14. Risse T., Knezevic P. - Data Storage Requirements for the Service Oriented Computing

15. Siegel J. - Developing in OMG's Model-Driven Architecture, OMG Staff Strategy Group

16. Trickovic I., Liu K. - Web Services Choreography, Position Paper for the first W3C Web Services Choreography Working Group Meeting, March, 2003.

17. Vernadat, F.B. - Enterprise Modeling and Integration: Principles and Applications. Chapman \& Hall. London, 1996. 